\title{
Reanimação de bebês prematuros moderados e tardios em sala de parto: fatores associados
}

Resuscitation of moderate and late preterm babies in the delivery room: associated factors Reanimación de bebés prematuros moderados y tardíos en sala de parto: factores relacionados

\author{
Maira Helena Muraro Descovi ${ }^{1}$ ic hitps://orcid.org/0000-0002-4451-5669 \\ Leonardo Bigolin Jantsch ${ }^{2}$ ic https://orcid.org/0000-0002-4571-183X, \\ Natana da Rosa ${ }^{2}$ ic https://orid.org/0000-0002-2000-1292. \\ Jaquiele Jaciara Kegler ${ }^{2}$ if https://orcid.org/0000-0003-0001-9564 \\ Eliane Tatsch Neves ${ }^{2}$ id hitps://orcid.org/0000-0002-1559-9533
}

\section{Como citar:}

Descovi MH, Jantsch LB, Rosa N, Kegler JJ, Neves ET. Reanimação de bebês prematuros moderados e tardios em sala de parto: fatores associados. Acta Paul Enferm. 2020;33:APE20180134.

DOI

http://dx.doi.org/10.37689/actaape/2020A00134

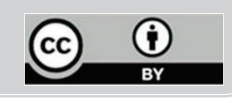

Descritores Nascimento prematuro; Salas de parto; Enfermagem neonatal

Keywords

Cardiopulmonary resuscitation; Infant, newborn; Premature birth; Delivery rooms; Neonatal nursing

\section{Descriptores}

Reanimación cardiopulmonar; Recién nacido; Nacimiento prematuro; Salas de parto; Enfermería

neonatal

Submetido 11 de Julho de 2018

Aceito

18 de Novembro de 2019

Autor correspondente

Maira Helena Muraro Descovi

E-mail: mairahelena@hotmail.com

\section{Resumo}

Objetivo: Verificar as variáveis obstétricas e neonatais relacionadas à necessidade de reanimação de recémnascidos (RN) prematuros moderados e tardios em sala de parto.

Métodos: Estudo transversal que incluiu 151 RN prematuros moderados e tardios de um hospital universitário do sul do Brasil. Participaram do estudo, todos os RN moderados e tardios, que nasceram no período de maio de 2016 a maio de 2017. A coleta aconteceu por meio dos prontuários dos participantes, utilizando instrumento próprio para coleta de dados. Os resultados foram apresentados por meio de frequências, comparação de frequência [Qui-Quadrado] para análise entre a variável dependente [necessidade de reanimação] e as independentes. 0 estudo seguiu as recomendações éticas.

Resultados: Os fatores obstétricos associados a necessidade de reanimação em RN moderados e tardios foram a gestação de risco $(p=0,007)$, intercorrências durante 0 parto $(p=0,031)$, cesariana $(p=0,005)$ e amniorrexe prematura $(p=0,01)$. Quanto a associação dos fatores neonatais, destaca-se as desproporções de peso para idade gestacional $(p<0,001)$, a menor idade gestacional $(p<0,001)$ e a malformação fetal $(p=0,047)$, como fatores relacionados a necessidade de reanimação.

Conclusão: Para população de prematuros moderados e tardios, fatores como amniorrexe prematura, menor idade gestacional e intercorrências gestacionais e no parto são fatores relacionados a necessidade de reanimação. Reconhecer esses fatores contribui para a gestão do cuidado nas salas de parto.

\section{Abstract}

Objective: To verify the obstetric and neonatal variables related to the need for resuscitation of moderate and late premature NBs in the delivery room.

Methods: A cross-sectional study that included 151 moderate and late preterm NBs from a university hospital in southern Brazil. All moderate and late NBs born from May 2016 to May 2017 participated in the study. The collection took place through the participants' medical records, using their own data collection instrument. Results were presented using frequencies, frequency comparison [Chi-Square] for analysis between the dependent variable [need for resuscitation] and the independent ones. The study followed ethical recommendations.

Results: The obstetric factors associated with the need for resuscitation in moderate and late NBs were risk pregnancy $(p=0.007)$, complications during delivery $(p=0.031)$, cesarean section $(p=0.005)$ and premature amniorrhexis $(p=0.01)$. Regarding the association of neonatal factors, we highlight the disproportion of weight for gestational age $(p<0.001)$, lower gestational age $(p<0.001)$ and fetal malformation $(p=0.047)$ as factors related to the need for resuscitation. 
Conclusion: For the population of moderate and late preterm NBs, factors such as premature amniorrhexis, early gestational age and gestational and delivery complications are factors related to the need for resuscitation. Recognizing these factors contributes to delivery room care management.

\section{Resumen}

Objetivo: Verificar las variables obstétricas y neonatales relacionadas con la necesidad de reanimación de recién nacidos (RN) prematuros moderados y tardíos en sala de parto.

Métodos: Estudio transversal que incluyó 151 RN prematuros moderados y tardíos de un hospital universitario del sur de Brasil. En el estudio participaron todos los RN moderados y tardíos que nacieron en el período de mayo de 2016 a mayo de 2017. La recolección se realizó a través de las historias clínicas de los participantes, con un instrumento propio para la recolección de datos. Los resultados se presentaron por medio de frecuencias, comparación de frecuencia (ji cuadrado) para el análisis entre la variable dependiente (necesidad de reanimación) y las independientes. El estudio cumplió las recomendaciones éticas.

Resultados: Los factores obstétricos relacionados con la necesidad de reanimación de RN moderados y tardíos fueron embarazo de riesgo ( $p=0,007$ ), complicaciones durante el parto $(p=0,031)$, cesárea $(p=0,005)$ y rotura prematura de membranas $(p=0,01)$. Respecto a la relación de los factores neonatales, se destacan la desproporción de peso para la edad gestacional $(p<0,001)$, la menor edad gestacional $(p<0,001)$ y la malformación fetal $(p=0,047)$, como factores relacionados con la necesidad de reanimación.

Conclusión: En la población de prematuros moderados y tardíos, factores como rotura prematura de membranas, menor edad gestacional y complicaciones gestacionales y en el parto están relacionados con la necesidad de reanimación. Reconocer esos factores contribuye para el manejo del cuidado en las salas de parto.

\section{Introdução}

O Recém-Nascido (RN) de alto risco é definido como aquele que apresenta risco de morbimortalidade maior que a média, associado às condiçóes de adaptação à vida extrauterina. Como RN de alto risco têm-se os prematuros, considerados aqueles nascidos com Idade Gestacional (IG) menor que 37 semanas, ${ }^{(1)}$ podendo ser subclassificados em prematuros extremos (até 28 semanas), muito prematuros (de 28 a menos de 32 semanas) e prematuros moderados a tardios (de 32 a 36 semanas e 6 dias). ${ }^{(2)}$

Estima-se que a cada ano nasçam no mundo 15 milhóes de prematuros, com até $12 \%$ destes nascimentos em países de baixa renda, dos quais um milhão morre devido a complicaçôes no parto. Além disso, aqueles que sobrevivem podem sofrer com algum tipo de sequela neurológica, auditiva, visual, de déficit de aprendizagem, entre outras, as quais podem acompanhá-los durante toda a vida. ${ }^{(2)}$

Existe uma preocupação mundial quando se refere à prematuridade, em especial a prematuros extremos ou muito prematuros, visto a fragilidade clínica imediata bem como as sequelas da sobrevida desses dentro das unidades de terapia intensiva. Contudo, uma parcela de prematuros muito presente nos serviços de saúde e que vem crescendo nos últimos anos, devido às cesáreas eletivas e complicaçôes obstétricas, são os moderados e tardios. Considera-se como prematuro moderado, o $\mathrm{RN}$ que apresenta de 32 a 33 semanas e 6 dias de IG e tardio, o de 34 a 36 semanas e 6 dias. ${ }^{(3)}$
Esses RN representam cerca de $10 \%$ do total de nascidos vivos no Brasil e sáo considerados "quase a termo" por alguns profissionais de saúde. Entretanto, constituem-se em uma clientela imatura em diferentes aspectos e, por vezes, apresentam maior necessidade de hospitalização relacionada à dificuldade respiratória, apneia, hipoglicemia, hipotermia, dificuldade de alimentação, icterícia, entre outras, bem como aspectos relacionados à adaptação extrauterina imediata. ${ }^{(1,4,5)}$

Essa necessidade de adaptação extrauterina maior em prematuros já é conhecida mundialmente e os dados do Programa de Reanimação Neonatal da Sociedade Brasileira de Pediatria confirmam que a necessidade de reanimaçáo neonatal é maior em $\mathrm{RN}$ pré-termo.${ }^{(6)}$ No Brasil, a cada ano nascem aproximadamente 300.000 crianças que requerem suporte para iniciar e manter a respiração, além daqueles prematuros que precisam de oxigenoterapia em sala de parto. ${ }^{(7)}$

A reanimação neonatal ou Ressuscitação Cardiopulmonar (RCP) na sala de parto ocorre com a recepçáo do RN e avaliaçáo da sua vitalidade. Neste caso, preconiza-se a atuação e tomada de decisão no "minuto de ouro", ou seja, avaliação da respiração e frequência cardíaca nos primeiros 60 segundos de vida, para definir a necessidade de manobras de reanimação neonatal, ${ }^{(6)}$ realizadas por médico ou enfermeiro capacitado, com treinamento teórico-prático, como preconizado pela portaria N. 371, de 7 de maio de 2014 do Ministério da Saúde. ${ }^{(7)}$ Entre as manobras de reanimação neonatal em sala de parto, destaca-se que cerca de um em 
cada dez RN necessita de ventilação com pressão positiva para iniciar a respiração, um em cada cem, precisa de intubaçáo e/ou compressão cardíaca e, ainda, um em cada mil, requer intubação, compressão cardíaca, associadas ao uso de medicamentos. ${ }^{(8)}$

Nesse sentido, justifica-se a realização deste estudo em virtude da grande demanda, representada por quase $90 \%$ dos partos prematuros ${ }^{(9)}$ atendidos nos serviços de saúde bem como pela importância de uma adequada identificação dos fatores predisponentes e pela tomada de decisáo em sala de parto, no intuito de minimizar possíveis sequelas ao $\mathrm{RN}$. ${ }^{(6)}$ Ainda, estudos voltados a população de RN moderados e tardios é incipiente e recente na literatura nacional e internacional, o que acaba por gerar uma lacuna no conhecimento científico. ${ }^{(10)}$

Desta forma, este estudo objetivou verificar as variáveis obstétricas e neonatais relacionadas à necessidade de reanimação de prematuros moderados e tardios em sala de parto.

\section{Métodos}

Trata-se de um estudo transversal, desenvolvido a partir do banco de dados do projeto matricial, intitulado "Condiçóes de saúde de prematuros moderados e tardios no primeiro ano de vida". Este objetivou analisar as condiçôes de saúde desses prétermos no primeiro ano de vida, por meio de um estudo misto.

O cenário foi um hospital de médio porte e alta complexidade da região sul do Brasil. A coleta de dados do projeto matricial ocorreu no período de 15 de maio de 2016 a 14 de maio de 2017. Foram incluídos no estudo todos os $\mathrm{RN}$ prematuros moderados e tardios, ou seja, com idade gestacional entre 32 a 33 semanas e 6 dias e 34 a 36 semanas e 6 dias $^{(3)}$, em que os responsáveis residiam no município sede do hospital.

A coleta de dados foi realizada pelo pesquisador e auxiliares de pesquisa devidamente capacitados, por meio de visita diária ao Centro Obstétrico (CO) para identificação dos participantes da pesquisa. A seguir, foi feita a busca dos mesmos na unidade de internação ou na unidade de terapia intensiva para o preenchimento do formulário com os dados do prontuário do RN.

Foi utilizado um formulário próprio, elaborado para este estudo e devidamente testado, contemplando variáveis obstétricas e neonatais, as quais foram preenchidas por meio do acesso aos prontuários. As variáveis maternas estudadas foram realização de pré-natal, gestação de risco e quais as condiçôes associadas a esta, intercorrências no parto e tipo de parto. Enquanto as variáveis neonatais consistiram em idade gestacional, relação peso $\mathrm{x}$ idade gestacional, sexo, Apgar do primeiro e quinto minuto, necessidade de internação em Unidade de Terapia Intensiva Neonatal (UTIN), dias de internação, necessidade de reanimação e a descrição das manobras de RCP utilizadas em sala de parto.

Os dados foram digitados e categorizados em um banco de dados no programa Epi-info ${ }^{\circledast}$ (versão 6.0), com dupla digitação independente. Após a verificação de erros e inconsistências na digitação, a análise dos dados foi realizada no programa estatístico Predictive Analytics Software (PASW Statistics" da SPSS Inc., Chicago, USA), versão 18.0. Os testes do Qui-quadrado e Exato de Fisher foram utilizados para associação de variáveis com os desfechos, sim ou não para necessidade de reanimação. Considerou-se com nível de significância quando $\mathrm{p}<0,05$. O banco de dados está constituído por 151 RN prematuros moderados e tardios.

Para a utilização do banco de dados foi solicitado uma autorização ao pesquisador responsável e encaminhado ao Comitê de Ética em Pesquisa da instituiçáo uma emenda ao projeto matricial, sendo aprovado sob o número do Certificado de Apresentação para Apreciação Ética (CAAE): 53898916.9.0000.5346 e número de parecer: 2.167.071. No projeto matricial, foram seguidas as recomendaçôes éticas e aplicado o termo de consentimento livre e esclarecido (TCLE) aos responsáveis, no momento da internação hospitalar.

\section{Resultados}

Participaram do estudo 151 prematuros moderados e tardios, dos quais 28 (18,5\%) eram moderados e 123 
(81,5\%) tardios. Dos $151 \mathrm{RN}$ participantes do estudo, $41(27,2 \%)$ necessitaram de RCP em sala de parto. A tabela 1 apresenta os fatores obstétricos associados à necessidade de reanimação neonatal em sala de parto.

Tabela 1. Variáveis obstétricas associadas à necessidade de reanimação de recém-nascidos moderados e tardios em sala de parto

\begin{tabular}{|c|c|c|c|c|}
\hline \multirow[b]{2}{*}{ Variáveis } & \multicolumn{3}{|c|}{ Necessidade de reanimação } & \multirow[b]{2}{*}{$p$-value } \\
\hline & $\begin{array}{l}\text { Sim } \\
\mathrm{n}(\%)\end{array}$ & $\begin{array}{l}\text { Não } \\
n(\%)\end{array}$ & $\begin{array}{l}\text { Total } \\
\mathrm{n}(\%)\end{array}$ & \\
\hline Intercorrência no parto & & & & $0,031^{*}$ \\
\hline Sim & $7(50,0)$ & $7(50,0)$ & $14(9,3)$ & \\
\hline Não & $34(24,8)$ & $103(75,2)$ & $137(90,7)$ & \\
\hline Pré-natal & & & & $0,642^{*}$ \\
\hline Sim & $27(27,3)$ & $72(72,7)$ & $99(65,6)$ & \\
\hline Não & $14(26,9)$ & $38(73,1)$ & $52(34,4)$ & \\
\hline Gestação de risco & & & & $0,007^{\star}$ \\
\hline Sim & $31(35,2)$ & $57(64,8)$ & $88(58,3)$ & \\
\hline Não & $10(15,9)$ & $53(84,1)$ & $63(41,7)$ & \\
\hline Incompetência cervical & & & & $0,481^{\star \star}$ \\
\hline Sim & $1(16,7)$ & $5(83,3)$ & $6(4,0)$ & \\
\hline Não & $40(27,6)$ & $105(72,4)$ & $145(96,0)$ & \\
\hline Sangramento no $1^{\circ} \mathrm{e} / \mathrm{ou} 2^{\circ}$ trimestre & & & & $0,190^{\star \star}$ \\
\hline Sim & $0(0,0)$ & $6(100,0)$ & $6(4,0)$ & \\
\hline Não & $41(28,3)$ & $104(71,7)$ & $145(96,0)$ & \\
\hline Descolamento de placenta & & & & $0,073^{*}$ \\
\hline Sim & $7(46,7)$ & $8(53,3)$ & $15(9,9)$ & \\
\hline Não & $34(25,0)$ & $102(75,0)$ & $136(90,1)$ & \\
\hline Amniorrexe prematura & & & & $0,01^{*}$ \\
\hline Sim & $7(14,3)$ & $42(85,7)$ & $49(32,5)$ & \\
\hline Não & $34(33,3)$ & $68(66,7)$ & $102(67,5)$ & \\
\hline Polidrâmnio/Oligoidrâmnio & & & & $0,593^{\star \star}$ \\
\hline $\operatorname{Sim}$ & $2(22,2)$ & $7(77,8)$ & $9(6,0)$ & \\
\hline Não & $39(27,5)$ & $103(72,5)$ & $142(94,0)$ & \\
\hline Gemelaridade & & & & $0,427^{\star \star}$ \\
\hline $\operatorname{Sim}$ & $4(22,2)$ & $14(77,8)$ & $18(11,9)$ & \\
\hline Não & $37(27,8)$ & $96(72,2)$ & $133(88,1)$ & \\
\hline Pré-eclâmpsia & & & & $0,227^{\star}$ \\
\hline Sim & $12(33,3)$ & $24(66,7)$ & $36(23,8)$ & \\
\hline Não & $29(25,2)$ & $86(74,8)$ & $115(76,2)$ & \\
\hline Malformação fetal & & & & $0,047^{\star \star}$ \\
\hline Sim & $4(66,6)$ & $2(33,3)$ & $6(4,0)$ & \\
\hline Não & $37(25,5)$ & $108(74,5)$ & $145(96,0)$ & \\
\hline RCIU & & & & $0,500^{\star \star}$ \\
\hline Sim & $2(33,3)$ & $4(66,6)$ & $6(4,0)$ & \\
\hline Não & $39(26,9)$ & $106(73,1)$ & $145(96,0)$ & \\
\hline Partos prematuros prévios & & & & $0,319^{*}$ \\
\hline Sim & $5(35,7)$ & $9(64,3)$ & $14(9,3)$ & \\
\hline Não & $36(26,3)$ & $101(73,7)$ & $137(90,7)$ & \\
\hline Doença materna & & & & $0,102^{*}$ \\
\hline Sim & $6(46,2)$ & $7(53,8)$ & $13(8,6)$ & \\
\hline Não & $35(25,4)$ & $103(74,6)$ & $138(91,4)$ & \\
\hline
\end{tabular}

RCIU - Restrição de Crescimento Intrauterino; *Qui-quadrado; ** Teste Exato de Fisher

Quanto às variáveis obstétricas, destaca-se que é, significativamente mais frequente, reanimar prematuros moderados e tardios quando houver alguma intercorrência no parto $(\mathrm{p}=0,031)$. Entre as inter- corrências, as mais recorrentes foram hemorragia materna $(30,8 \%)$, seguida de hipertensão $(15,4 \%)$ e de hipotensão (15,4\%). Também, a reanimação neonatal em sala de parto foi significativamente mais frequente em filhos que nasceram de gestaçôes de risco $(\mathrm{p}=0,007)$ e que apresentaram amniorrexe prematura de placenta $(p=0,01)$. Os motivos de gestação de risco mais frequentes foram doença hipertensiva específica da gestação, gemelaridade, diabetes mellitus gestacional, gemelaridade associada à doença hipertensiva específica da gestação e partos prematuros prévios. As demais variáveis obstétricas não apresentaram diferença significativa entre os que necessitaram reanimação e aqueles que não foram reanimados. Quanto as variáveis neonatais que tiveram relação com a necessidade de reanimação, destaca-se a tabela 2 .

Tabela 2. Variáveis neonatais associadas à necessidade de reanimação de recém-nascidos moderados e tardios em sala de parto

\begin{tabular}{|c|c|c|c|c|}
\hline \multirow{3}{*}{ Variáveis } & \multicolumn{3}{|c|}{ Necessidade de reanimação } & \multirow{3}{*}{$p$-value } \\
\hline & $\operatorname{Sim}$ & Não & Total & \\
\hline & $n(\%)$ & $\mathrm{n}(\%)$ & $\mathrm{n}(\%)$ & \\
\hline Idade Gestacional & & & & $<0,001^{\star}$ \\
\hline Moderado & $20(71,4)$ & $8(28,6)$ & $28(18,5)$ & \\
\hline Tardio & $21(17,1)$ & $102(82,9)$ & $123(81,5)$ & \\
\hline Relação Peso X IG & & & & $<0,001^{\star}$ \\
\hline AlG & $22(19,8)$ & $89(80,2)$ & $111(73,5)$ & \\
\hline PIG ou GIG & $19(47,5)$ & $21(52,5)$ & $40(26,5)$ & \\
\hline Sexo & & & & $0,570^{*}$ \\
\hline Masculino & $18(25,0)$ & $54(75,0)$ & $72(47,7)$ & \\
\hline Feminino & $23(29,1)$ & $56(70,9)$ & $79(52,3)$ & \\
\hline Parto & & & & $0,005^{\star}$ \\
\hline Vaginal & $5(11,4)$ & $39(88,6)$ & $44(29,1)$ & \\
\hline Cesárea & $36(33,6)$ & $71(66,4)$ & $107(70,9)$ & \\
\hline Apgar do 1" minuto & & & & $<0,001^{\star \star}$ \\
\hline$\geq 7$ & $15(12,3)$ & $107(87,7)$ & $122(80,8)$ & \\
\hline$<7$ & $26(89,7)$ & $3(10,3)$ & $29(19,2)$ & \\
\hline Apgar do 5" minuto & & & & $<0,001^{\star *}$ \\
\hline$\geq 7$ & $34(23,8)$ & $109(76,2)$ & $143(94,7)$ & \\
\hline$<7$ & $8(100,0)$ & $0(0,0)$ & $8(5,3)$ & \\
\hline Necessitou de internação em UTIN & & & & $<0,0001^{*}$ \\
\hline Sim & $33(53,2)$ & $29(46,8)$ & $62(41,1)$ & \\
\hline Não & $8(9,0)$ & $81(91,0)$ & $89(58,9)$ & \\
\hline Dias de internação na UTIN (n=62) & & & & $0,042^{*}$ \\
\hline 1-3 dias & $8(38,1)$ & $13(61,9)$ & $21(33,9)$ & \\
\hline 4-10 dias & $7(41,2)$ & $10(58,8)$ & $17(27,4)$ & \\
\hline$>10$ dias & $18(75,0)$ & $6(25,0)$ & $24(38,7)$ & \\
\hline Dias de internação hospitalar & & & & $<0,0001^{*}$ \\
\hline $1-3$ dias & $8(13,3)$ & $52(86,7)$ & $60(39,7)$ & \\
\hline 4-10 dias & $9(18,0)$ & $41(82,0)$ & $50(33,1)$ & \\
\hline$>10$ dias & $24(58,5)$ & $17(41,5)$ & $41(27,2)$ & \\
\hline
\end{tabular}

IG - Idade Gestacional; PIG - Pequeno para a Idade Gestacional; AIG - Adequado para a Idade Gestacional; GIG - Grande para a Idade Gestacional; UTIN - Unidade de Terapia Intensiva Neonatal. ${ }^{*}$ Qui-quadrado; ** Teste Exato de Fisher. 
Quanto às variáveis neonatais, 71,4\% dos prematuros moderados e $17,1 \%$ dos prematuros tardios necessitaram reanimação, essa diferença foi significativamente maior para a população de moderados $(\mathrm{p}<0,001)$, quando comparados aos tardios. RN que nasceram por parto cesáreo $(70,9 \%)$, apresentaram maior necessidade de reanimação quando comparados àqueles nascidos por parto vaginal $(\mathrm{p}=0,005)$. Quanto à necessidade de reanimação e a relação de peso e IG, prevaleceu reanimação neonatal entre RN PIG ou GIG, quando comparados aos que nasceram com peso AIG $(\mathrm{p}<0,001)$. Também, quanto menor o Apgar no primeiro e quinto minuto de vida maior a necessidade de reanimação em sala de parto $(\mathrm{p}<0,001)$, Apgar menor que 7 no primeiro e quinto minuto confirmou a necessidade de reanimação. Além disso, todos os RN com Apgar menor que 7 no quinto minuto de vida precisaram de suporte especializado para adaptação extrauterina.

Dos $\mathrm{RN}$ reanimados em sala de parto, $53,2 \%$ precisaram ser internados em UTIN, sendo que a maioria deles permaneceu mais de dez dias internados em UTIN (75\%) e hospitalizados (58,5\%). Percebe-se que a necessidade de reanimação neonatal está diretamente associada à necessidade de internação em UTIN.

A tabela 3 apresenta as manobras de reanimação utilizadas em sala de parto.

Tabela 3. Manobras de reanimação utilizadas em recémnascidos prematuros moderados e tardios em sala de parto

\begin{tabular}{lc}
\hline Manobras de reanimação & n(\%) \\
\hline Máscara facial + oxigenioterapia & $13(31,7)$ \\
Ventilação Pulmonar c/ pressão positiva & $7(17,1)$ \\
Ventilação Pulmonar c/ pressão positiva + oxigênio & $16(39,0)$ \\
Intubação orotraqueal & $2(4,9)$ \\
Intubação orotraqueal + compressão cardíaca & $1(2,4)$ \\
Intubação orotraqueal + uso de fármaco vasoativo & $2(4,9)$ \\
\hline
\end{tabular}

As manobras de reanimação mais utilizadas em sala de parto foram a Ventilaçáo Pulmonar com pressão positiva mais oxigênio, seguida da oferta de oxigênio por máscara facial.

\section{Discussão}

Os riscos obstétricos durante a gestação representam maiores riscos para a necessidade de reanimação neonatal em sala de parto. Estudo que elencou riscos gestacionais semelhantes aos deste estudo (distúrbios hipertensivos, diabetes gestacional, amniorrexe, placenta prévia, restrição de crescimento intrauterino, polidrâmnio/oligoidrâmnio) concluiu que à exceçáo da idade materna avançada, os demais riscos representaram maior chance de reanimação em sala de parto entre RN a termo e prematuros. (11) Neste estudo, com a população de prematuros moderados e tardios, as gestaçóes de risco e aquelas com amniorrexe prematura, apresentaram frequência maior para necessidade de reanimação.

Um terço dos nascimentos prematuros estão associados a amniorrexe prematura de membranas. Embora isso cause um aumento nos casos de morbidade neonatal e na necessidade de internação em UTIN, principalmente, por infecçóes neonatais, ela náo se mostrou associada a necessidade de RCP. ${ }^{(12)} \mathrm{Na}$ população de a termos, ela não foi fator de risco para necessidade de reanimação, ${ }^{(13)}$ o que pode ser característica específica dessa população de moderados e tardios. Ainda, em contraponto aos achados desse estudo, pesquisadores brasileiros que analisaram risco para reanimação em população de RN pré-termos e a termos, destacam que apenas 4,2\% dos RN de mães com amniorrexe necessitaram de suporte especializado para adaptação extrauterina. ${ }^{(14)}$ Desta forma, mais estudos precisam ser desenvolvidos com a temática, a fim de identificar se a necessidade de reanimação pode ter como uma das causas a amniorrexe prematura.

Já os casos de descolamento prematuro de placenta representaram 46,7\% dos casos de necessidade de reanimação em sala de parto. Em estudo desenvolvido em Santa Catarina, evidenciou-se que $85,7 \%$ e $80 \%$ dos $\mathrm{RN}$ com Apgar baixo no primeiro e quinto minuto de vida, respectivamente, eram prematuros oriundos de gestaçóes onde houve descolamento prematuro de placenta, o que se assemelha a esta pesquisa. ${ }^{(15)}$

Dentre os tipos de parto, a cesárea é associada à maior necessidade de reanimação neonatal avançada. (16) Também, embora a Organização Mundial da Saúde aponte a cesárea como fator positivo para salvar vidas maternas e neonatais, os índices de cirurgias estão acima do esperado (mais de 10\%). Deste modo, acabam sendo realizadas cesáreas desnecessárias, que podem causar danos tanto para a mãe quanto para o RN. ${ }^{(17,18)}$ 
Quanto aos fatores neonatais, autores corroboram que o baixo peso e a prematuridade têm relação direta com o resultado da avaliação da vitalidade do $\mathrm{RN}$ no momento do parto. A íntima relação da prematuridade e o baixo peso destacam a vulnerabilidade do prematuro e suas possíveis complicaçóes posteriores, com as baixas avaliaçóes de Apgar no primeiro e quinto minuto de vida e da necessidade de reanimação em sala de parto. ${ }^{(13,18)}$

Cabe destacar que a necessidade de reanimação em sala de parto foi significativamente mais frequente nos moderados quando comparados aos tardios. Estudos corroboram com os achados e destacam que a necessidade de reanimação é inversamente proporcional a idade gestacional e/ou peso ao nascer e, que no Brasil, anualmente cerca de 300.000 nascidos necessitam ser reanimados em sala de parto. Na população de a termos, destaca-se que $10 \%$ dos nascidos necessitam de reanimação, relacionado as principais causas maternas/obstétricas, como as doenças maternas prévias e a idade materna, bem como motivos fetais, como malformaçóes ou polioligodramia. ${ }^{(19,20)}$

Igualmente, no Reino Unido, em um estudo de caso controle com prematuros moderados, tardios e RN a termos, observou-se maior prevalência de necessidade de reanimação neonatal entre os prétermos moderados $(36,9 \%)$ quando comparados aos prematuros tardios e RN a termos $(14,5 \%$ e $7,4 \%$ consecutivamente). ${ }^{(18)}$ Da mesma forma, os $\mathrm{RN}$ prematuros moderados necessitaram mais de suporte respiratório, como por exemplo, ventilação mecânica e ventilação com pressão positiva, o que corrobora com este estudo.

Também, da mesma forma que um estudo desenvolvido em Portugal, este evidenciou maior necessidade de reanimação entre os RN prematuros PIG e/ ou GIG. Demonstrando que os prematuros tardios apresentam duas vezes mais chances de necessitarem de reanimação avançada quando são PIG ou GIG. ${ }^{\text {(21) }}$

Outro fator associado a reanimação de prematuros moderados e tardios foi a presença de malformaçóes neonatais. Da mesma forma que como apontam as diretrizes de reanimação desenvolvidas pela Sociedade Brasileira de Pediatria, nesta pesquisa evidencia-se a prevalência de reanimação em recém-nascidos prematuros quando esses apresentam algum tipo de malformação, sejam elas descoberta antenatal ou não, dando ênfase a necessária realização do pré-natal. ${ }^{(6)}$

Destaca-se a importância do desenvolvimento de um pré-natal adequado para fins de identificação precoce de possíveis riscos neonatais e demais agravos no momento do nascimento/recepção do RN em sala de parto. Nessa perspectiva, cabe destacar que nesse estudo, mesmo náo apresentando diferença significativa, cerca de $34 \%$ das gestantes não tinham realizado número de consultas adequadas de pré-natal, o que confere risco para parto prematuro. ${ }^{(22)}$

Quanto à necessidade de internação em UTIN e dias de permanência, notou-se maior incidência de internação nos pré-termos moderados e tardios que necessitaram de algum tipo de ressuscitação em sala de parto $(53,2 \%)$, sendo que a maioria dessas crianças teve longa permanência sob cuidados intensivos (75\%). Esse achado corrobora com um estudo de coorte, que concluiu que RN prematuros moderados que necessitaram de RCP em sala de parto, permanecem mais dias hospitalizados, apresentaram maior necessidade de suporte respiratório aos 28 dias de vida e tiveram retardo no início da alimentação oral. ${ }^{(16)}$ Neste estudo, 58,5\% dos RN ficaram mais de 10 dias hospitalizados. Além disso, nota-se que os RN que apresentaram Apgar menor que 7 no quinto minuto de vida em $100 \%$ das vezes necessitaram de reanimação neonatal em sala de parto, consequentemente, justifica-se os altos números de internação sob cuidados intensivos, embora o valor do boletim de Apgar não indique isoladamente reanimação neonatal. ${ }^{(13)}$

Para tanto, reconhecer os intervenientes e fatores relacionados à necessidade de reanimação em sala de parto permite a qualificação das equipes de saúde, a fim de que as manobras de reanimação e a sistemática aconteçam o mais rápido possível e possam ser minimizados os dados/riscos ao RN. Reconhecer na prática do enfermeiro em sala de parto, os fatores associados a reanimação bem como as manobras mais utilizadas na população de prematuros moderados e tardios permite a gestão do cuidado de forma efetiva e singular, visto a ascensão dessa população nos serviços de saúde.

Este estudo apresentou como limitação o fato de ter analisado a reanimação neonatal em sala de 
parto de prematuros moderados e tardios somente oriundos do município sede do hospital. Visto que, o cenário da pesquisa foi um hospital referência em obstetrícia para a região central do estado.

\section{Conclusão}

Quanto às variáveis obstétricas e neonatais relacionadas à necessidade de reanimação de prematuros moderados e tardios em sala de parto, pode-se concluir que RN moderados, PIG ou GIG e que apresentaram Apgar menor que 7 no $1^{\circ}$ e $5^{\circ}$ minuto de vida, possuem maior chance de serem reanimados. Destes, sua maioria necessitou internação em terapia intensiva neonatal bem como permaneceram por período maior hospitalizados, quando comparados aos que não foram reanimados. Quanto aos fatores obstétricos, é importante destacar que a amniorrexe prematura foi uma característica peculiar desse estudo, para essa população, a qual exige novas pesquisas que façam a relação da reanimação neonatal com esse fator de risco.

\section{Agradecimentos}

Financiamento de bolsas de Iniciação Científica na realização do estudo. Financiamento Institucional PIBIC/CNPQ (UFSM) e PROIC/HUSM.

\section{Colaborações}

Descovi MHM, Jantsch LB, Rosa N, Kegler JJ e Neves ET declaram que contribuíram com a concepção do estudo, análise e interpretação dados, redação do artigo, revisão critica relevante do conteúdo intelectual e aprovação da versão final a ser publicada.

\section{Referências}

1. World Health ICD-11 for Mortality and morbidity statistics (ICD-11 MMS) 2018 version [cited 2019 Sep 26], 11th revision. Geneva: WHO; 2018. Available from: https://icd.who.int/browse11/l-m/en
2. World Health Organization (WHO). Preterm birth. Fact sheet. Geneva: WHO; 2017. [ cited 2018 Mar 12]. Available from: http://www.who.int/ mediacentre/factsheets/fs363/es/

3. Engle WA. Recommendation for the definition of "late preterm" (nearterm) and the birth weight-gestational age classification system. Semin Perinatol. 2006;30(1): 2-7.

4. Guzmán AA, Fernández YG, Rodríguez NM, Matos AA. Recién nacidos pretérminos tardíos, un grupo de riesgo. Rev Cubana Pediatr. 2016; 88(2):144-55.

5. Berry AL, Samson K, Hanson C, Garg A, Su D. State populationbased changes in preterm delivery - impact of efforts to enforce recommendations to eliminate early elective deliveries [Meeting Abstract,136]. Pediatrics. 2018;142(1).

6. Sociedade Brasileira de Pediatria. Reanimação do recém-nascido z34 semanas em sala de parto: Diretrizes 2016 da Sociedade Brasileira de Pediatria [Internet]. São Paulo: Sociedade Brasileira de Pediatria; 2016. [citado 2019 Set 30]. Disponível em: http://www. sbp.com.br/reanimacao

7. Brasil. Ministério da Saúde. Portaria no. 371, de 7 de maio de 2014 Institui diretrizes para a organização da atenção integral e humanizada ao recém-nascido (RN) no Sistema Único de Saúde (SUS). Diário Oficial da União (DOU) - Seção I, N. 86, 8 de mai. 2014.

8. Sociedade Brasileira de Pediatria. Reanimação Neonatal em Sala de Parto: Documento Científico do Programa de Reanimação Neonatal da Sociedade Brasileira de Pediatria [Internet]. São Paulo: Sociedade Brasileira de Pediatria; 2013. 50p. [citado 2019 Set 30]. Disponível em: http://www.sbp.com.br/reanimacao

9. Delnord M, Zeitlin J. Epidemiology of late preterm and early term births-An international perspective. Semin Fetal Neonatal Med. 2019; 24(3):3-10.

10. Currie G, Dosani A, Premji SS, Reilly SM, Lodha AK, Young M. Caring for late preterm infants: public health nurses' experiences. BMC Nurs. 2018;17. Article number 16. doi: https://doi.org/10.1186/s12912018-0286-y

11. Yangthara B, Horrasith S, Paes B, Kitsommart R. Predictive factors for intensive birth resuscitation in a developing-country: A 5-year, singlecenter study. J Matern Fetal Neonatal Med. 2018:1-7.

12. Moreira ME, Pereira AP, Junior SC, Guinsburg R, de Almeida MF, Gama, $S G$, et al Factors associated with the use of supplemental oxygen or positive pressure ventilation in the delivery room, in infants born with a gestational age $\geq 34$ Weeks. The Author(s) Reproductive Health. 2016;13 (S3 Suppl):116.

13. Saraiva JP, Vogt SE, Rocha JS, Duarte ED, Simão DA. Association between maternal and neonatal factors and Apgar in usual risk neonates. Rev Rene. 2018;19:e3179.

14. Suárez GJ, Gutiérrez MM, Noorani RA, Gaibor IV. La rotura prematura de membranas pretérmino y su relación con las consecuencias maternas y perinatales. Acta Med Cent. 2016; 10(3):40-7.

15. Nunes RD, Bertuol E, Siqueira IR, Avaliação dos fatores associados aos resultados neonatais no descolamento prematuro de placenta. Arq Catarin Med. 2016 45(4):11-27.

16. Bajaj M, Natarajan G, Shankaran S, Wyckoff M, Laptook AR, Bell EF, et al. Delivery room resuscitation and short-term outcomes in moderately preterm infants. J Pediatr. 2018; 195:33-8.e2.

17. World Health Organization (WHO). WHO Declaration on Cessation Rates. [Internet] Genève: WHO; 2015. [cited 2017 Jun 12]. Available from: http://www.who.int/about/ licensing/copyright_form/ en/index. html 
18. Choudhary B, Choudhary Y, Pakhare AP, Mahto D, Chaturvedula L. Early neonatal outcome in caesarean section: A developing country perspective. Iran J Pediatr. 2017. 27(1):e7948.

19. Ersdal HL, Eilevstiønn J, Linde JE, Yeconia A, Mduma ER, Kidanto H, et al. Fresh stillborn and severely asphyxiated neonates share a common hypoxic-ischemic pathway. Int J Gynaecol Obstet. 2018;141(2):171180.

20. Perlman JM, Wyllie J, Kattwinkel J, Wyckoff MH, Aziz K, Guinsburg R, Kim HS, Liley HG, Mildenhall L, Simon WM, Szyld E, Tamura M, Velaphi S; Neonatal Resuscitation Chapter Collaborators. Part 7: Neonatal
Resuscitation: 2015 International Consensus on Cardiopulmonary Resuscitation and Emergency Cardiovascular Care Science With Treatment Recommendations (Reprint). Pediatrics. 2015;136 Suppl 2:S120-66.

21. Boyle EM, Johnson S, Manktelow B, Seaton SE, Draper ES, Smith LK, et al. Neonatal outcomes and delivery of care for infants born late preterm or moderately preterm: a prospective population-based study. Arch Dis Child Fetal Neonatal. 2015;100(6):479-85.

22. Lourenço N, Fernandes M, Gomes C, Resende C. Morbidade neonatal dos recém-nascidos pré-termo tardios comparados aos de termo precoce. Sci Med. 2017; 27(1):25876. 